\title{
Potencial antioxidante dos extratos de manjericão (Ocimum basilicum Lamiaceae) e orégano (Origanum vulgare Lamiaceae) em óleo de soja
}

\author{
PITARO, S.P.; FIORANI, L.V.; JORGE, N.*
}

Departamento de Engenharia e Tecnologia de Alimentos, Instituto de Biociências, Letras e Ciências Exatas, Universidade Estadual Paulista "Júlio de Mesquita Filho", Campus São José do Rio Preto, Rua Cristóvão Colombo, 2265, Jardim Nazareth, CEP: 15054-000, São José do Rio Preto-Brasil *njorge@ibilce.unesp.br

\begin{abstract}
RESUMO: O presente trabalho teve como objetivos avaliar o potencial antioxidante dos extratos de manjericão (Ocimum basilicum L.) e orégano (Origanum vulgare L.), e medir a estabilidade oxidativa do óleo de soja adicionado de ambos os extratos. Foram obtidos extratos de manjericão e orégano nas formas in natura e seco, a partir das folhas das especiarias submetidas às extrações aquosas, etanólicas e sequenciais por $30 \mathrm{~min}$, na proporção de 1:3 ( $\mathrm{m} / \mathrm{m}$, especiaria:solvente), sob agitação contínua e temperatura ambiente. Nos extratos foram determinados o valor de concentração eficiente $\left(\mathrm{CE}_{50}\right)$, a atividade antioxidante máxima $(\mathrm{AA})$ e a quantidade de compostos fenólicos totais (CFT). Os extratos de orégano e manjericão com maior atividade antioxidante foram aplicados no óleo de soja em concentrações que variaram de 250 a $2.000 \mathrm{mg} \mathrm{kg}^{-1}$, para avaliação da estabilidade oxidativa. Os resultados indicaram que os extratos etanólicos de manjericão in natura com $\mathrm{CE}_{50}=863,97 \mu \mathrm{g} \mathrm{mL}^{-1}, \mathrm{AA}=35,63 \%$ e CFT $=56,55 \mathrm{mg} \mathrm{g}^{-1}$ e orégano seco com $\mathrm{CE}_{50}=$ $415,82 \mu \mathrm{g} \mathrm{mL}^{-1}, \mathrm{AA}=48,42 \%$ e CFT $=147,96 \mathrm{mg} \mathrm{g}^{-1}$ foram os mais eficientes para serem aplicados ao óleo de soja na concentração de $2.000 \mathrm{mg} \mathrm{kg}^{-1}$, na qual apresentaram a maior estabilidade oxidativa, 21,8 e 15,1 horas, respectivamente. Com base na metodologia empregada e dentro das condições estudadas, concluiu-se que os extratos etanólicos de manjericão in natura e orégano seco revelaram potencial antioxidante quando aplicados em óleo de soja.
\end{abstract}

Palavras-chave: especiarias, extratos etanólicos, atividade antioxidante

\begin{abstract}
Potential of basil (Ocimum basilicum L.) and oregano (Origanum vulgare L.) extracts as antioxidant to soybean oil. This study aimed to evaluate the antioxidant potential of basil (Ocimum basilicum L.) and oregano (Origanum vulgare L.) extracts and to measure the oxidative stability of soybean oil added to both extracts. Basil and oregano extracts were obtained in fresh and dry forms from the leaves of spices subjected to aqueous, ethanol and sequential extraction for $30 \mathrm{~min}$ at a ratio of $1: 3(\mathrm{~m} / \mathrm{m}$, spice:solvent) and continuously shaken at room temperature. For the extracts, the value of effective concentration $\left(E C_{50}\right)$, the maximum antioxidant activity (AA) and the amount of total phenolic compounds (TFC) were obtained. The oregano and basil extracts that had higher antioxidant activity were applied to soybean oil at concentrations ranging from 250 to $2,000 \mathrm{mg} \mathrm{kg}^{-1}$, to evaluate the oxidative stability. The results indicated that the ethanol extracts of fresh basil with $E_{50}=863.97 \mathrm{mg} \mathrm{mL}^{-1}, \mathrm{AA}=35.63 \%$ and TFC $=56.55 \mathrm{mg} \mathrm{g}^{-1}$ and dry oregano with $\mathrm{EC}_{50}=415.82 \mathrm{mg} \mathrm{mL}^{-1}, \mathrm{AA}=48.42 \%$ and TFC $=147.96 \mathrm{mg} \mathrm{g}^{-1}$ were more efficient for application in soybean oil at a concentration of $2,000 \mathrm{mg} \mathrm{kg}^{-1}$, which showed the highest oxidative stability, 21.8 and 15.1 hours, respectively. Based on the employed methodology and under the studied conditions, it was concluded that the ethanol extracts of fresh basil and dry oregano showed antioxidant potential when applied in soybean oil.
\end{abstract}

Key words: spices, ethanol extracts, antioxidant activity

\section{INTRODUÇÃO}

O número de contribuições para os métodos de isolamento, técnicas, e testes de atividade antioxidante de origem vegetal tem aumentado significativamente nos últimos anos, tendo em vista que a oxidação é uma das principais causas de deterioração química, resultando na deterioração da

Recebido para publicação em 05/08/2011

Aceito para publicação em 12/04/2012

Rev. Bras. PI. Med., Botucatu, v.14, n.4, p.686-691, 2012. 
qualidade nutricional, cor, textura, sabor e segurança dos alimentos (Antolovich et al., 2002; Gülçin et al., 2007; Rosa et al., 2010).

A presença de antioxidantes pode reduzir ou prevenir a oxidação lipídica, apesar de terem a estabilidade reduzida quando expostos a altas temperaturas. Os antioxidantes sintéticos, butilhidroxianisol (BHA), butil-hidroxitolueno (BHT), galato de propila (GP) e terc-butilhidroquinona (TBHQ) são os mais utilizados (Haiying et al., 2008). Entretanto, o emprego dos antioxidantes sintéticos tem sido alvo de questionamentos quanto à inocuidade (Nobuyoki et al., 2003; Sasse et al., 2009).

Sobre o uso de antioxidantes naturais, importantes aspectos tecnológicos e nutricionais podem ser destacados, como a conservação da qualidade dos alimentos, principalmente nos ricos em gorduras poli-insaturadas podendo substituir os antioxidantes sintéticos; a preservação da saúde humana, ao minimizar danos oxidativos principalmente em algumas doenças e a presença de componentes bioativos, caracterizando os alimentos como funcionais (Lagouri \& Nisteropoulou, 2009; Silva et al., 2010).

Por estes motivos, muitas pesquisas têm sido realizadas com a finalidade de encontrar produtos naturais com atividade antioxidante, com destaque para as especiarias, pois podem ser adicionadas aos alimentos em várias formas, como especiarias inteiras ou como isolados dos extratos (Hossain et al., 2010).

As especiarias são ingredientes aromáticos e pungentes, portanto, o uso direto como antioxidantes é limitado. O procedimento de extração é determinado pelos tipos de compostos antioxidantes a serem extraídos. A seleção do procedimento de extração pode influenciar na concentração de antioxidante do material vegetal. Para compostos fenólicos e outros antioxidantes em materiais vegetais três técnicas de extração podem ser usadas, a extração com uso de solventes, a em fase sólida e a com uso de solventes a baixa pressão (extração supercrítica). É aconselhável para otimizar a extração, o uso de amostras secas, congeladas, ou liofilizadas, uma vez que alguns antioxidantes são instáveis e podem ser degradados pela ação da enzima em materiais de origem vegetal não desidratados (Suhaj, 2006).

Dentre as especiarias, o manjericão possui elevado poder antioxidante (Gülçin et al., 2007; Juntachote et al., 2007). Os compostos fenólicos responsáveis pela atividade antioxidante do manjericão estão presentes em ácidos fenólicos, como o ácido rosmarínico derivado do ácido caféico (Aguiyi et al., 2000; Melo et al., 2006). O orégano, especiaria com sabor altamente favorável aos consumidores de todo o mundo, também recebe destaque pelas propriedades antimicrobianas e antioxidantes (Yanishlieva et al., 2006) devido os compostos carvacróis, flavonóides e terpenos, tais como apigenina, dihidrocampferol e dihidroquercetina (Arcila-Lozano et al., 2004).

Desta forma, este estudo pode favorecer a descoberta de novas fontes de antioxidantes naturais com contribuição significativa no campo da saúde em nível mundial, encontrando substâncias eficazes e menos tóxicas para serem aplicadas em alimentos. Assim, o presente estudo teve por objetivo avaliar o potencial antioxidante dos extratos de manjericão e orégano isolados e quando aplicados em óleo de soja.

\section{MATERIAL E MÉTODO}

\section{Especiarias}

As especiarias, manjericão (Ocimum basilicum L.) e orégano (Origanum vulgare L.) in natura, foram coletados na cidade de São José do Rio Preto (SP). As exsicatas das especiarias foram realizadas no Departamento de Zoologia e Botânica da UNESP e as espécimes-testemunhas foram depositadas no herbário SJRP sob os números SJRP30006 e SJRP-30007.

Das especiarias foram retiradas as raízes, os talos e as folhas secas. Uma parte das folhas das especiarias foi disposta em bandeja e seca em estufa com circulação de ar forçada (marca Marconi, modelo MA033) a $40^{\circ} \mathrm{C}$, por 24 horas. Após a desidratação, o material foi triturado e passado em peneira de tyler 3,5 com abertura de 5,66 mm. Em seguida foi acondicionado em recipientes plásticos com barreira à luz e mantido sob congelamento até o momento da utilização. A parte restante das especiarias in natura foi utilizada para obtenção dos extratos in natura.

\section{Obtenção dos extratos}

Foram obtidos seis diferentes extratos para cada especiaria, sendo três deles da especiaria in natura, e o restante, da especiaria seca. Os solventes de extração utilizados foram água destilada e o etanol, usados de forma isolada e sequencial, dando origem aos extratos aquosos, etanólicos e sequenciais, os quais foram avaliados quanto às respectivas atividades antioxidantes.

Para obtenção dos extratos aquosos (in natura e seco), $25 \mathrm{~g}$ da especiaria foram adicionadas de $75 \mathrm{~mL}$ de água destilada. A mistura foi homogeneizada em liquidificador (marca Funkitchen, modelo WJ-6801R) por 30 minutos a velocidade de rotação média; em seguida, foi filtrada em bomba a vácuo separando-se o sobrenadante do precipitado. Procedeu-se então a remoção do solvente utilizado para obtenção do extrato, em evaporador rotativo (marca Quimis, modelo Q-344B2) sob pressão reduzida a $60^{\circ} \mathrm{C}$, com objetivo de determinar, por pesagem direta, o rendimento da extração. 
Para os extratos etanólicos (in natura e seco), foi feito o mesmo procedimento descrito para os extratos aquosos, porém, adicionando à especiaria $75 \mathrm{~mL}$ do solvente etanol e removendo-o posteriormente sob pressão reduzida à $40^{\circ} \mathrm{C}$, em evaporador rotativo.

A partir do precipitado obtido do extrato etanólico na filtração em bomba a vácuo (marca Tecnal, modelo TE058), foram obtidos os extratos sequenciais das especiarias in natura e secas. Neste precipitado foram adicionados $75 \mathrm{~mL}$ de água destilada, e, novamente, foi submetido ao processo de extração nas mesmas condições anteriormente explicitadas para os extratos aquosos.

\section{Atividade antioxidante}

A avaliação quantitativa da atividade antioxidante dos extratos das especiarias foi feita segundo o procedimento descrito por Brand-Williams et al. (1995), monitorando-se o consumo do radical livre 2,2-difenil-1-picrilhidrazil (DPPH ${ }^{\circ}$ ) pelos extratos, através da medida da absorbância de soluções de diferentes concentrações.

Preparou-se soluções metanólicas com concentração de $500 \mu \mathrm{g} \mathrm{mL}^{-1}$ de extrato aquoso da especiaria, soluções etanólicas de $500 \mu \mathrm{g} \mathrm{mL}^{-1}$ de extrato etanólico da especiaria e soluções metanólicas de $500 \mu \mathrm{g} \mathrm{mL}^{-1}$ de extrato sequencial da especiaria. Cada amostra destas soluções $(0,3 \mathrm{~mL})$ foi adicionada a $2,7 \mathrm{~mL}$ de solução de $\mathrm{DPPH}^{\bullet}(40 \mu \mathrm{g}$ $\left.\mathrm{mL}^{-1}\right)$ em diferentes concentrações $(5,10,25,50,125$ e $250 \mu \mathrm{g} \mathrm{mL}^{-1}$ ). Após o tempo de reação de 30 minutos, a absorbância foi lida em $515 \mathrm{~nm}$ e convertida em porcentagem de atividade antioxidante.

O controle foi feito com $2,7 \mathrm{~mL}$ de $\mathrm{DPPH}^{\bullet} \mathrm{e}$ o branco realizado com $0,3 \mathrm{~mL}$ de solução do extrato e 2,7 mL do solvente utilizado na extração (água ou etanol), para cada concentração.

Esta metodologia permite a determinação da concentração eficiente $\left(\mathrm{CE}_{50}\right)$, também chamada de concentração inibitória, definida como a quantidade de antioxidante necessária para decrescer a concentração inicial de $\mathrm{DPPH}^{\bullet}$ em $50 \%$. A CE $\mathrm{C}_{50}$ foi determinada a partir de uma curva exponencial de primeira ordem, obtida ao plotar na abscissa as concentrações da amostra $\left(\mu \mathrm{g} \mathrm{mL}^{-1}\right)$ e na ordenada, a porcentagem de atividade antioxidante.

\section{Compostos fenólicos totais}

A quantificação de compostos fenólicos totais foi determinada por espectrofotometria, por meio do reagente de Folin-Ciocauteau, segundo a metodologia descrita por Singleton \& Rossi (1965). Este método baseia-se na redução dos ácidos fosfomolíbdico e fosfotúngstico em solução alcalina. A cor azul produzida pela redução do reagente Folin-Ciocauteau pelos fenólicos é medida no espectrofotômetro (marca
Shimadzu, modelo UV mini 1240). Para quantificação foi utilizada uma curva padrão de ácido gálico em concentrações de 0 a $500 \mathrm{mg} \mathrm{L}^{-1}$.

Neste procedimento, pipetou-se $100 \mu \mathrm{L}$ da solução de extrato em tubos de ensaio e adicionou-se $500 \mu \mathrm{L}$ do reagente de Folin-Ciocauteau. Em seguida, adicionou-se $1,5 \mathrm{~mL}$ de solução saturada de carbonato de sódio $20 \%(\mathrm{~m} / \mathrm{v})$ e $6 \mathrm{~mL}$ de água destilada.

Essa mistura permaneceu em repouso por 2 horas em temperatura ambiente, e a absorbância foi determinada a $765 \mathrm{~nm}$. Os resultados foram expressos em miligramas de equivalentes de ácido gálico por grama de extrato (mg EAG g ${ }^{-1}$ ). Os reagentes e o padrão empregados nesta determinação foram provenientes da marca Sigma-Aldrich, St.Louis, MO.

\section{Estabilidade oxidativa}

Os extratos de manjericão e orégano mais eficazes foram aplicados em óleo de soja para determinação da estabilidade oxidativa seguindo o método proposto pela AOCS (2009). Foram testadas concentrações que variaram de 250 a $2.000 \mathrm{mg} \mathrm{kg}^{-1}$ utilizando-se o Rancimat (marca Metrohm, modelo 743), equipamento baseado na determinação da condutividade elétrica dos produtos voláteis da degradação. Por este método, uma curva de condutividade elétrica versus tempo é automaticamente registrada com o decorrer da reação e do teste, sendo o período de indução expresso em horas.

O teste foi realizado a $100^{\circ} \mathrm{C}$, com fluxo de ar de $20 \mathrm{~L} \mathrm{~h}^{-1}$, com $3 \mathrm{~g}$ de amostra e volume de água destilada de $60 \mathrm{~mL}$ nos frascos contendo os eletrodos.

\section{Análise estatística}

Para as amostras submetidas ao ensaio de estabilidade oxidativa foram considerados os seguintes fatores: tratamentos (OS + EM e OS + EO) e concentrações $(0,250,500,750,1.000,1.500$ e $2.000 \mathrm{mg} \mathrm{kg}^{-1}$ ). Os resultados da estabilidade oxidativa, em três repetições, foram submetidos à análise de variância em esquema fatorial $2 \times 7$, no delineamento inteiramente casualizado (Banzatto \& Kronka, 2006), e ao teste de Tukey para as médias a $5 \%$ foram obtidos através do programa ESTAT Sistema para Análises Estatísticas, versão 2.0.

\section{RESULTADO E DISCUSSÃO}

Os rendimentos dos diferentes extratos de manjericão e orégano, calculados por pesagem direta após a remoção do solvente utilizado em cada uma das extrações estão apresentados na Tabela 1.

Os extratos de manjericão e orégano in natura apresentaram baixos rendimentos ao final da extração, com valores que oscilaram de 2,96 a 4,01\% e 3,14 a $5,81 \%$, respectivamente, em relação aos

Rev. Bras. PI. Med., Botucatu, v.14, n.4, p.686-691, 2012. 
TABELA 1. Rendimentos (\%) dos extratos de manjericão e orégano.

\begin{tabular}{cl|c}
\hline Extratos & & Rendimentos \\
\hline \multirow{2}{*}{ Manjericão } & Aquoso & 4,01 \\
in natura & Etanólico & 3,98 \\
& Sequencial & 2.96 \\
\hline \multirow{2}{*}{ Manjericão } & Aquoso & 5,71 \\
seco & Etanólico & 7,48 \\
& Sequencial & 15.51 \\
\hline \multirow{2}{*}{ Orégano } & Aquoso & 3,14 \\
in natura & Etanólico & 5,81 \\
& Sequencial & 4,03 \\
\hline \multirow{2}{*}{ Orégano } & Aquoso & 12,75 \\
seco & Etanólico & 12,07 \\
& Sequencial & 6,29 \\
\hline
\end{tabular}

extratos de manjericão e orégano secos, o que significa que a utilização de material previamente desidratado pode maximizar a eficiência do processo de extração. Dentre os extratos de manjericão seco, a extração sequencial dos solventes utilizados proporcionou o maior rendimento (15,51\%), entretanto, dentre os extratos de orégano seco, o aquoso e o etanólico alcançaram aproximadamente o dobro de rendimento em relação ao sequencial. A extração dos compostos está diretamente relacionada à composição do produto e a polaridade dos mesmos, havendo grande diversidade em relação ao material e solvente utilizado (Turkmen et al., 2006; Rockenbach et al., 2008).

Os valores de $\mathrm{CE}_{50}$, obtidos por regressão linear são calculados pela redução de $50 \%$ da concentração inicial de $\mathrm{DPPH}^{\bullet} \mathrm{e}$, quanto menor o valor, maior a atividade antioxidante dos compostos analisados.

Os compostos fenólicos totais foram quantificados colorimetricamente nos extratos de manjericão e orégano, e, para quantificação foi utilizada uma curva padrão de ácido gálico em concentrações de 0 a $500 \mathrm{mg} \mathrm{L}^{-1}$. O coeficiente de determinação da curva analítica foi de 0,9986.

$\mathrm{Na}$ Tabela 2 constam os percentuais máximos de atividade antioxidante $(A A)$, os valores de $\mathrm{CE}_{50}$, e as medidas de compostos fenólicos totais (CFT) para os extratos de manjericão e orégano.

De acordo com os dados apresentados na Tabela 2, observa-se que os percentuais máximos de atividade antioxidante dos extratos etanólicos de manjericão in natura e seco, 35,63 e 32,52\%, respectivamente, superaram os percentuais encontrados para os demais extratos de manjericão. Apesar da proximidade entre estes valores, o valor de $\mathrm{CE}_{50}, 863,97$ $\mu \mathrm{g} \mathrm{mL}^{-1}$, e a concentração de CFT, $56,55 \mathrm{mg} \mathrm{g}^{-1}$, do extrato etanólico de manjericão in natura, comprovam o quanto este extrato foi mais eficiente. Dentre os extratos de orégano, observa-se que a porcentagem máxima de atividade antioxidante do extrato etanólico de orégano seco $(48,42 \%)$ foi maior que a do aquoso $(28,08 \%)$ e que do sequencial $(36,46 \%)$, assim como também foi superior aos de orégano in natura (14,30-40,38\%), portanto, verifica-se que o extrato etanólico de orégano seco apresentou além do maior percentual de atividade antioxidante, menor valor de $\mathrm{CE}_{50}\left(415,82 \mu \mathrm{g} \mathrm{mL}^{-1}\right) \mathrm{e}$ maior quantidade de compostos fenólicos totais $(147,96$ $\mathrm{mg} \mathrm{g}^{-1}$ ) em relação aos demais extratos de orégano, sendo considerado o mais eficiente para ser aplicado no óleo de soja.

TABELA 2. Médias de AA (\%), $\mathrm{CE}_{50}\left(\mu \mathrm{g} \mathrm{mL}^{-1}\right)$ e CFT $\left(\mathrm{mg} \mathrm{g}^{-1}\right)$ dos extratos de manjericão e orégano.

\begin{tabular}{|c|c|c|c|c|c|}
\hline Extratos & & AA & $C E_{50}$ & CFT & Correlação* \\
\hline \multirow{3}{*}{$\begin{array}{l}\text { Manjericão } \\
\text { in natura }\end{array}$} & Aquoso & $10,34^{\mathrm{K}}$ & $10.837,37^{\mathrm{A}}$ & $12,46^{\mathrm{L}}$ & \multirow{3}{*}{0,99} \\
\hline & Etanólico & $35,63^{\mathrm{E}}$ & $863,97^{\mathrm{G}}$ & $56,55^{\mathrm{F}}$ & \\
\hline & Sequencial & $14,12^{\prime}$ & $2.526,54^{\mathrm{E}}$ & $18,40^{\prime}$ & \\
\hline \multirow{3}{*}{$\begin{array}{l}\text { Manjericão } \\
\text { Seco }\end{array}$} & Aquoso & $11,41^{J}$ & $6.538,53^{C}$ & $18,19^{J}$ & \multirow{3}{*}{0,84} \\
\hline & Etanólico & $32,52^{\mathrm{F}}$ & $1.315,74^{\mathrm{F}}$ & $20,66^{\mathrm{H}}$ & \\
\hline & Sequencial & $8,15^{\mathrm{L}}$ & $6.716,58^{\mathrm{B}}$ & $13,56^{\mathrm{K}}$ & \\
\hline \multirow{3}{*}{$\begin{array}{l}\text { Orégano } \\
\text { in natura }\end{array}$} & Aquoso & $14,30^{\mathrm{H}}$ & $2.999,18^{D}$ & $24,00^{G}$ & \multirow{3}{*}{0,99} \\
\hline & Etanólico & $36,09^{D}$ & $596,69^{\prime}$ & $112,92^{D}$ & \\
\hline & Sequencial & $40,38^{\mathrm{B}}$ & $470,86^{\mathrm{K}}$ & $147,61^{\mathrm{B}}$ & \\
\hline \multirow{3}{*}{$\begin{array}{l}\text { Orégano } \\
\text { Seco }\end{array}$} & Aquoso & $28,08^{G}$ & $622,89^{H}$ & $89,45^{\mathrm{E}}$ & \multirow{3}{*}{0,99} \\
\hline & Etanólico & $48,42^{A}$ & $415,82^{\mathrm{L}}$ & $147,96^{\mathrm{A}}$ & \\
\hline & Sequencial & $36,46^{\mathrm{C}}$ & $578,01^{J}$ & $114,24^{\mathrm{C}}$ & \\
\hline
\end{tabular}

A, B... (coluna): médias seguidas de mesma letra maiúscula não diferem pelo teste de Tukey $(p>0,05)$. ${ }^{*} A A$ e CFT 
Diante dos resultados para os diferentes extratos de manjericão e orégano observa-se que os extratos etanólicos de manjericão in natura e de orégano seco apresentaram maior eficiência quanto à capacidade antioxidante entre os extratos analisados, diferença evidenciada estatisticamente entre as demais concentrações analisadas conforme teste de Tukey $(p<0,05)$.

Em estudos realizados por Del Ré \& Jorge (2011) os resultados demonstraram grande variação no teor de compostos fenólicos totais em oleoresinas de tomilho e orégano, 64,77 e $25,93 \mathrm{mg}$ de equivalente de ácido gálico por 100 gramas de oleoresina, respectivamente. Alguns autores encontraram elevadas correlações entre atividade antioxidante e compostos fenólicos em extratos de especiarias (Maksimoviæ et al., 2005; Carvalho Filho et al., 2006).

A estabilidade oxidativa, em horas, fornecida pelo Rancimat, para o óleo de soja adicionado de extrato etanólico das especiarias (manjericão in natura e orégano seco) em diferentes concentrações, está apresentada na Tabela 3.

Independente do tipo de extrato observa-se que os valores da estabilidade oxidativa aumentaram com o aumento das concentrações do extrato, não demonstrando efeito pró-oxidante. Em geral, as mesmas características são encontradas na literatura para a ação antioxidante dos extratos naturais, ou seja, a tendência é o aumento da atividade antioxidante, conforme o aumento da concentração de extrato (Haiying etal., 2008).

Com base nos resultados da estabilidade oxidativa, obtidos pela regressão polinomial, entre as concentrações de extrato de gengibre avaliadas $(0 \mathrm{a}$ $2.500 \mathrm{mg} \mathrm{kg}^{-1}$ ), a mais efetiva foi de $2.500 \mathrm{mg} \mathrm{kg}^{-1}$, para retardar da oxidação lipídica (Andreo \& Jorge, 2011). Luzia \& Jorge (2010) estudaram o comportamento do óleo de soja adicionado de extrato de sementes de limão galego em diferentes concentrações ( 0 a 2.500 $\mathrm{mg} \mathrm{kg}^{-1}$ ) e concluíram que a concentração de 2.400 $\mathrm{mg} \mathrm{kg}^{-1}$ foi a que conferiu melhor estabilidade oxidativa ao óleo.

Com base nos resultados da estabilidade oxidativa, verifica-se que os extratos de manjericão e orégano na concentração de $2.000 \mathrm{mg} \mathrm{kg}^{-1}$, com médias de 21,8 e 15,1 horas, respectivamente, mostraram maior eficiência na proteção do óleo de soja, diferença evidenciada estatisticamente entre as demais concentrações analisadas, conforme teste de Tukey $(p<0,05)$.

O aumento do período de indução por meio da adição de um antioxidante tem sido relacionado à eficiência do antioxidante, que é expresso como fator de proteção ou índice antioxidante, isto é, a razão entre o período de indução de um óleo na presença do antioxidante e o período de indução do mesmo óleo na ausência do antioxidante (Holasova et al., 2006). Na Tabela 4 estão apresentados os fatores de proteção para as concentrações testadas dos extratos das especiarias.

Observa-se que os extratos de ambas as especiarias (manjericão e orégano) na concentração de $2.000 \mathrm{mg} \mathrm{kg}^{-1}$ foram mais eficientes em retardar a oxidação, em relação às demais concentrações, pois quanto maior o valor do fator de proteção, melhor a atividade antioxidante (Elizable et al., 1992).

Ainda é possível observar pelos valores contidos na Tabela 4, que os extratos das especiarias protegeram o óleo em retardar a oxidação, no entanto,

TABELA 3. Médias da estabilidade oxidativa (horas) do óleo de soja adicionado dos extratos etanólicos de manjericão e orégano em diferentes concentrações $\left(\mathrm{mg} \mathrm{kg}^{-1}\right)$.

\begin{tabular}{c|c|c|c|c|c|c|c}
\hline \multirow{2}{*}{ Tratamentos } & \multicolumn{7}{|c}{ Concentrações } \\
\cline { 2 - 8 } & $\mathbf{0}$ & $\mathbf{2 5 0}$ & $\mathbf{5 0 0}$ & $\mathbf{7 5 0}$ & $\mathbf{1 . 0 0 0}$ & $\mathbf{1 . 5 0 0}$ & $\mathbf{2 . 0 0 0}$ \\
\hline OS + EM & $10,3^{\mathrm{fA}}$ & $11,3^{\mathrm{eB}}$ & $11,4^{\mathrm{eB}}$ & $13,6^{\mathrm{dA}}$ & $15,8^{\mathrm{CA}}$ & $18,7^{\mathrm{bA}}$ & $21,8^{\mathrm{aA}}$ \\
OS + EO & $10,3^{\mathrm{fA}}$ & $12,7^{\mathrm{eA}}$ & $13,0^{\mathrm{dA}}$ & $13,4^{\mathrm{CA}}$ & $13,5^{\mathrm{bcB}}$ & $13,6^{\mathrm{bB}}$ & $15,1^{\mathrm{aB}}$ \\
\hline
\end{tabular}

OS + EM: óleo de soja com extrato de manjericão; OS + EO: óleo de soja com extrato de orégano. a, b... (linha): médias seguidas de mesma letra minúscula não diferem pelo teste de Tukey $(p>0,05)$. A, B... (coluna): médias seguidas de mesma letra maiúscula não diferem pelo teste de Tukey $(p>0,05)$.

TABELA 4. Média dos fatores de proteção para as concentrações $\left(\mathrm{mg} \mathrm{kg}^{-1}\right)$ dos extratos de manjericão e orégano aplicados em óleo de soja.

\begin{tabular}{c|c|c|c|c|c|c}
\hline \multirow{2}{*}{ Tratamentos } & \multicolumn{7}{|c}{ Concentrações } \\
\cline { 2 - 7 } & $\mathbf{2 5 0}$ & $\mathbf{5 0 0}$ & $\mathbf{7 5 0}$ & $\mathbf{1 . 0 0 0}$ & $\mathbf{1 . 5 0 0}$ & $\mathbf{2 . 0 0 0}$ \\
\hline OS + EM & $1,10^{\mathrm{fB}}$ & $1,11^{\mathrm{eB}}$ & $1,32^{\mathrm{dA}}$ & $1,53^{\mathrm{CA}}$ & $1,82^{\mathrm{bA}}$ & $2,12^{\mathrm{aA}}$ \\
OS + EO & $1,19 \mathrm{eA}$ & $1,21^{\mathrm{dA}}$ & $1,25^{\mathrm{CB}}$ & $1,26 \mathrm{bcB}$ & $1,27^{\mathrm{bB}}$ & $1,41^{\mathrm{aB}}$ \\
\hline
\end{tabular}

OS + EM: óleo de soja com extrato de manjericão; OS + EO: óleo de soja com extrato de orégano. a, b... (linha): médias seguidas de mesma letra minúscula não diferem pelo teste de Tukey $(p>0,05)$. A, B... (coluna): médias seguidas de mesma letra maiúscula não diferem pelo teste de Tukey $(p>0,05)$. 
o extrato de manjericão apresentou-se mais eficaz quando comparado ao de orégano e a melhor concentração a ser aplicada foi de $2.000 \mathrm{mg} \mathrm{kg}^{-1}$ para ambos os extratos, diferença evidenciada estatisticamente entre as demais concentrações analisadas conforme teste de Tukey $(p<0,05)$.

\section{CONCLUSÃO}

Os extratos de manjericão e orégano estudados apresentaram potencial antioxidante, uma vez que os valores de atividade antioxidante e de compostos fenólicos totais foram maiores nos extratos etanólicos de manjericão in natura e orégano seco. Estes extratos de especiarias proporcionaram maior estabilidade oxidativa, quando aplicados em óleo de soja na concentração de $2.000 \mathrm{mg} \mathrm{kg}^{-1}$. Porém, novos estudos são necessários para avaliar seus reais potenciais antioxidantes no óleo de soja.

\section{AGRADECIMENTO}

Ao Conselho Nacional de Desenvolvimento Científico e Tecnológico (CNPq), pelas bolsas de Iniciação Científica e Produtividade em Pesquisa.

\section{REFERÊNCIA}

AGUIYI, J.C. et al. Hyypoglycaemic activity of Ocimum gratissimum in rats. Fitoterapia, v.71, n.4, p.444-6, 2000. ANDREO, D.; JORGE, N. Capacidade antioxidante e estabilidade oxidativa de Gengiber officinal. UNOPAR Científica. Ciências Biológicas e da Saúde, v.13, p.33-7, 2011. ANTOLOVICH, M. et al. Methods for testing antioxidant activity. Analyst, v.127, n.1, p.183-98, 2002.

AOCS - AMERICAN OIL CHEMISTS SOCIETY. Official methods and recommended practices of the American Oil Chemists' Society. Champaign: AOCS, 2009.

ARCILA-LOZANO, C.C. et al. El orégano: propiedades, composición y actividad biológica de sus componentes. Archivos Latinoamericanos de Nutrición, v.54, n.1, p.10011, 2004

BANZATTO, D.A.; KRONKA, S.N. Experimentação agrícola. Jaboticabal: FUNEP, 2006. 237p.

BRAND-WILLIAMS, W. et al. Use of a free radical method to evaluate antioxidant activity. Lebensmittel - Wissenschaft und Technologie, v.28, n.1, p.25-30, 1995.

CARVALHO FILHO, J.L.S. et al. Influence of the harvesting time, temperature and drying period on basil (Ocimum basilicum L.) essential oil. Revista Brasileira de Farmacognosia, v.16, n.1, p.24-30, 2006.

DEL RÉ, P.V.; JORGE, N. Antioxidant potential of oregano (Oreganum vulgare L.), basil (Ocimum basilicum L.) and thyme (Thymus vulgaris L.): application of oleoresins in vegetable oil. Ciência e Tecnologia de Alimentos, v.31, n.4, p.955-9, 2011.

ELIZABLE, B.E. et al. Antioxidative action of maillard reactions volatiles: influence of maillard solution level. Journal of the American Oil Chemists' Society, v.69, n.3, p.331-4, 1992.
GÜLÇIN, I. et al. Determination of antioxidant and radical scavenging activity of basil (Ocimum basilicum L.) assayed by different methodologies. Phytotherapy Research, v.21, n.4, p.354-61, 2007.

HAIYING, T. et al. The effect of natural and synthetic antioxidants on the oxidative stability of biodiesel. Journal of the American Oil Chemists' Society, v.85, n.4, p.373-82, 2008.

HOLASOVA, M. et al. Buckwheat - the source of antioxidant activity in functional foods. Food Research International, v.35, n.2, p.207-11, 2006.

HOSSAIN, M.V. et al. Effect of drying method on the antioxidant capacity of six Lamiaceae herbs. Food Chemistry, v.123, n.1, p.85-91, 2010.

JUNTACHOTE, T. et al. Antioxidative effect of added dried holy basil and its ethanolic extracts on susceptibility of cooked ground pork to lipid oxidation. Food Chemistry, v.100, n.1, p.129-35, 2007.

LAGOURI, V.; NISTEROPOULOU, E. Antioxidant properties of $O$. onites, $T$. vulguris and $O$. basilicum species grown in Greece and their total phenol and rosmarinic acid content. Journal of Food Lipids, v.16, n.2, p.484-98, 2009.

LUZIA, D.M.M.; JORGE, J. Potencial antioxidante de extratos de sementes de limão (Citrus limon). Ciência e Tecnologia de Alimentos, v.30, n.2, p.489-93, 2010.

MAKSIMOVIÆE, Z. et al. Polyphenol contents and antioxidant activity of Maydis stigma extracts. Bioresource Technology, v.96, p.873-7, 2005.

MELO, A.S. et al. Influence of the harvesting time, temperature and drying period on basil (Ocimum basilicum L.) essential oil. Revista Brasileira de Farmacognosia, v.16, n.1, p.24-30, 2006.

NOBUYOKI, I. et al. Antioxidants: carcinogenic and chemopreventive properties. Encyclopedia of Cancer, v.1, n.1, p.89-101, 2003.

ROCKENBACH, I.I. et al. Influência do solvente no conteúdo total de polifenóis, antocianinas e atividade antioxidante de extratos de bagaço de uva (Vitis vinifera) variedades Tannat e Ancelota. Ciência e Tecnologia de Alimentos, v.28, p.23844, 2008

ROSA, E. et al. Flavonoides e atividade antioxidante em Palicourea rigida Kunth, Rubiaceae. Revista Brasileira de Farmacognosia, v.20, n.4, p.484-88, 2010.

SASSE, A. et al. Effect of natural and synthetic antioxidants on the oxidative stability of cooked, frozen pork patties.

Journal of Food Science, v.74, n.1, p.30-5, 2009.

SILVA, M.L.C. et al. Compostos fenólicos, carotenóides e atividade antioxidante em produtos vegetais. Ciências Agrárias, v.31, n.3, p.669-82, 2010.

SINGLETON, V.L.; ROSSI, J.A.J. Colorimetry of total phenolics with phosphomolybdic-phosphotungstic acid reagents. American Journal of Enology and Viticulture, v.16, n.3, p.144-58, 1965.

SUHAJ, M. Spice antioxidants isolation and their antiradical activity: a review. Journal of Food Composition and Analysis, v.19, n.4, p.531-7, 2006.

TURKMEN, N. et al. Effects of extraction solvents on concentration and antioxidant activity of black and black mate tea polyphenols determined by ferrous tartrate and Folin-Ciocalteu methods. Food Chemistry, v.99, n.4, p. 835-41, 2006.

YANISHLIEVA, N.V. et al. Natural antioxidants from herbs and spices. European Journal of Lipid Science and Technology, v.108, n.9, p.776-93, 2006. 